\title{
FINITE GROUP ACTIONS ON SIEGEL MODULAR SPACES
}

\author{
K. F. LAI AND RONNIE LEE
}

\begin{abstract}
The theory of nonabelian cohomology is used to show that the set of fixed points of a finite group acting on a Siegel modular space is a union of Shimura varieties
\end{abstract}

\section{INTRODUCTION}

Let $g$ be a finite group of automorphisms of the symplectic group $\operatorname{Sp}_{2 n}(\mathbb{R})$ which leaves invariant the maximal compact subgroup $U_{n}(\mathbb{C})$ and let $\Gamma$ be a torsion free arithmetic subgroup of $\operatorname{Sp}_{2 n}(\mathbb{Q})$ which is invariant under $g$. Denote by $\mathfrak{G}_{n}$ the Siegel upper half space of degree $n$, i.e.,

$$
\mathfrak{G}_{n}=\left\{Z \in M_{n}(\mathbb{C}) \mid Z={ }^{t} Z, \operatorname{Im} Z>0\right\} .
$$

Then $g$ induces an action on the locally symmetric space $\mathfrak{G}_{n} / \Gamma$. We denote by $\left(\mathfrak{G}_{n} / \Gamma\right)^{\mathscr{g}}$ the set of fixed-points of $g$ in $\mathfrak{G}_{n} / \Gamma$. Gottschiling [2,3] gave a very explicit description of the fixed-point sets of $g$ in $\mathfrak{G}_{2} / \Gamma$. The set of components of $\left(\mathfrak{G}_{n} / \Gamma\right)^{\mathcal{g}}$ is in one-to-one correspondence with the nonabelian cohomology set $H^{1}(g, \Gamma)$ (cf. [8]). The arithmetic nature of these components is determined by the image in $H^{1}\left(g, \operatorname{Sp}_{2 n}(\mathbb{Q})\right)$ under the natural map

$$
H^{1}(g, \Gamma) \rightarrow H^{1}\left(g, \operatorname{Sp}_{2 n}(\mathbb{Q})\right)
$$

Using the results of Satake [7] on symplectic representations, we describe in $\S 3$ $H^{1}\left(g, \mathrm{Sp}_{2 n}(\mathbb{Q})\right)$ in terms of the character table of $g$. The use of nonabelian cohomology to study the fixed-point set was first discovered by Rohlfs. In $\S 1$, we summarize his theory with minor modification to fit our situation. In the next section, we relate the nonabelian cohomology with the representation theory of $g$. The authors would like to thank the referee for helpful comments.

\section{FIXED-POINT COMPONENTS}

1.1. Let $G$ be a connected semisimple algebraic group defined over $\mathbb{Q}$, let $K$ be a fixed maximal compact subgroup of the group $G(\mathbb{R})$ of the real points of $G$, and $X=K \backslash G(\mathbb{R})$ the associated symmetric space.

Let $g$ be a finite group of automorphisms of $G(\mathbb{R})$ which keeps the maximal compact subgroup $K$ invariant. Then there exists an induced action of $g$ on the symmetric space $X=K \backslash G(\mathbb{R})$ by isometries. Suppose we are given a

Received by the editors January 3, 1991 and, in revised form, August 7, 1991.

1980 Mathematics Subject Classification (1985 Revision). Primary 20G05, 14K10, 57 S25.

Key words and phrases. Fixed point set, Shimura variety. 
torsion free, $g$-stable, arithmetic subgroup $\Gamma$ of $G$. Then $g$ acts on the quotient space $X / \Gamma$. Denote by $(X / \Gamma)^{\mathscr{g}}$ the fixed-point set of this action.

1.2. A cocycle in $Z^{1}(g, \Gamma)$ is a map, $s \mapsto b_{s}$, of $g$ to $\Gamma$ such that $b_{s t}=$ $b_{s} \cdot s\left(b_{t}\right)$, where ${ }^{s}\left(b_{t}\right)$ represents the action of $s(s \in g)$ on the element $b_{t}$. The cohomology class of $b_{s}$ is denoted by $\left[b_{s}\right]$.

1.3. Given an element $b=\left[b_{s}\right]$ in $H^{1}(g, \Gamma)$, we define a new action of $g$ on $G(\mathbb{R})$ by means of the formula: $A \mapsto{ }^{\bar{s}} A=b_{s} \cdot{ }^{s}(A) \cdot b_{s}^{-1}$. There is also an action of $g$ on $X$,

$$
{ }^{\bar{s}} x={ }^{s} x \cdot b_{s}^{-1}, \quad x \in X, s \in g,
$$

and this is compatible with the action of $g$ on the group $G(\mathbb{R})$,

$$
{ }^{\bar{s}}(x \cdot A)={ }^{\bar{s}} x \cdot{ }^{\bar{s}} A .
$$

We will refer to these as the $g$-action twisted by the cocycle $b$ and will use the notation $g(b)$ to denote the new automorphism group. We write $X(b)$ for the fixed-point set of $g(b)$ in $X$. Let $\Gamma(b)$ be the set of $g(b)$-invariant elements of $\Gamma$. We have a natural embedding $X(b) / \Gamma(b) \rightarrow X / \Gamma$ and we denote by $F(b)$ the image of this embedding. Clearly, this is a part of the fixed-point set $(X / \Gamma)^{\mathcal{g}}$. If two cocycles $b$ and $b^{\prime}$ are cohomologous to each other, then the fixed-point sets $X(b), X\left(b^{\prime}\right)$ are $\Gamma$-conjugate to each other, and so $F(b)=F\left(b^{\prime}\right)$.

1.4. The connected components of $(X / \Gamma)^{\mathscr{g}}$ are indexed by the cohomology set $H^{1}(g, \Gamma)$.

Proposition. The fuxed-point set $(X / \Gamma)^{\mathcal{g}}$ is the disjoint union of the connected totally geodesic submanifolds $F(b)$,

$$
(X / \Gamma)^{\mathscr{g}}=\bigcup_{b} F(b)
$$

where $b$ runs through $H^{1}(g, \Gamma)$.

The proof follows the same lines as that of Proposition 1.3 of Rohlfs [6]. We omit the proof.

1.5. We shall need the following lemma, the proof of which is the same as Rohlfs [6, Lemma 1.4].

Lemma. Let $K$ be the fixed maximal compact subgroup in $G(\mathbb{R})$, and let $g$ be a finite subgroup in Aut $G(\mathbb{R})$ which keeps the subgroup $K$ invariant. Then the inclusion $i: K \hookrightarrow G(\mathbb{R})$ induces a bijection in cohomology $i: H^{1}(g, K) \rightarrow$ $H^{1}(g, G(\mathbb{R}))$.

1.6. Given an element $b$ in $H^{1}(g, \Gamma)$, we denote by $b_{\infty}$ the image of $b$ in $H^{1}(g, G(\mathbb{R}))$ under the natural induced map $H^{1}(g, \Gamma) \rightarrow H^{1}(g, G(\mathbb{R}))$. Clearly if $b_{\infty}=b_{\infty}^{\prime}$, then $X(b)$, and $X\left(b^{\prime}\right)$ are isomorphic to each other under the translation of an element in $G(\mathbb{R})$. Thus it is enough to study $X(b)$ according to the classification in $H^{1}(g, G(\mathbb{R}))$.

By the above lemma (1.5), we may assume that $b$ is an element in $H^{1}(g, K)$ and we get an exact sequence of pointed sets

$$
1 \rightarrow H^{0}(g(b), K) \rightarrow H^{0}(g(b), G(\mathbb{R})) \rightarrow H^{0}(g(b), X) \rightarrow 1 .
$$


From the definition $H^{0}(g(b), X)$ is the same as the $g(b)$-fixed-point set $X(b)$ in $X$, and $H^{0}(g(b), K), H^{0}(g(b), G(\mathbb{R}))$ are subgroups in $K$ and $G(\mathbb{R})$ respectively fixed by $g(b)$, and the sequence (1.6.1) can be viewed as a fibration sequence of spaces

$$
1 \rightarrow K^{\mathscr{P}^{(b)}} \rightarrow G(\mathbb{R})^{\mathscr{f}^{(b)}} \rightarrow X(b) \rightarrow 1 .
$$

We have $K^{\mathscr{g}^{(b)}}=K \cap G(\mathbb{R})^{(b)}$ and that $K^{\mathscr{f}^{(b)}}$ is the maximal compact subgroup in $G(\mathbb{R})^{\mathscr{f}(b)}$ and $X(b)$ is the symmetric space associated to $G(\mathbb{R})^{\mathfrak{g}^{(b)}}$. Summing up, we have

1.6.4. Proposition. A connected component $F(b)$ of the fixed-point set $(X / \Gamma)^{g(b)}$ of the locally symmetric space $X / \Gamma$ is again locally symmetric. With the notation defined as in the above paragraph, $F(b)$ is the quotient of the symmetric space $X(b)=H^{0}(g(b), K) \backslash H^{0}(g(b), G(\mathbb{R}))$ associated to the Lie group $H^{0}(g(b), G(\mathbb{R}))$ and it has the arithmetic subgroup $H^{0}(g(b), \Gamma)$ as its covering transformation group,

$$
F(b)=H^{0}(g(b), K) \backslash H^{0}(g(b), G(\mathbb{R})) / H^{0}(g(b), \Gamma) .
$$

\section{NONABELIAN COHOMOLOGY}

2.1. To proceed further, we need an explicit description of the nonabelian cohomology $H^{i}(g, G), i=0,1$, in terms of $g$.

Let us assume that $G$ is semisimple and $\operatorname{rk}_{\mathbb{Q}} G>0$ and that every element in $g$ is an inner automorphism, given via a homomorphism $\rho: g \rightarrow \operatorname{Inn}(G)$. It is then enough to consider the central extension of groups

$$
1 \rightarrow Z(G) \rightarrow G \rightarrow \operatorname{Inn}(G) \rightarrow 1 .
$$

Using the homomorphism $\rho: g \rightarrow \operatorname{Inn}(G)$, we can pull back the above central extension to obtain a covering group $\hat{g}$ of $g$ together with a commutative diagram of group homomorphisms

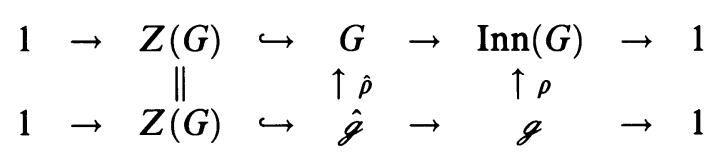

Here $\hat{\rho}$ is a homomorphism of $\hat{g}$ to $G$ which covers our original homomorphism $\rho: g \rightarrow \operatorname{Inn}(G)$, and restricts to the identity map on the center $Z(G)$.

In general, we consider the set $\operatorname{Hom}_{Z(G)}(\hat{g}, G)$ of all group homomorphisms $\theta: \hat{g} \rightarrow G$ which restricts to the identity on the center. Two such homomorphisms $\theta: \hat{g} \rightarrow G, \theta^{\prime}: \hat{g} \rightarrow G$ are said to be equivalent if they differ by the conjugation with an element $x$ in $G$. The set of all these equivalence classes of homomorphisms will be denoted by $\operatorname{Hom}_{Z(G)}(\hat{g}, G) / G$.

2.2. Proposition. There is a one-to-one-correspondence

$$
H^{1}(\mathrm{~g}, G) \stackrel{\cong}{\rightarrow} \operatorname{Hom}_{Z(G)}(\hat{g}, G) / G .
$$

This follows immediately from the exact sequence

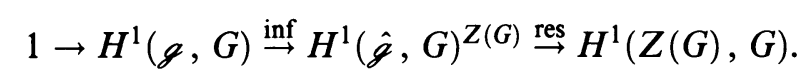


2.3. For $b$ in $H^{1}(g, G)$. Let $\theta_{b}$ be image of $b$ under the isomorphism of Proposition 2.2. As in (1.3), we can twist the action $\rho: g \rightarrow \operatorname{Aut}(G)$ of $g$ on $G$ by an element $b$ in $H^{1}(g, G)$ using the formula

$$
A \mapsto \theta_{b}(u) \rho(u)(A) \theta_{b}(u)^{-1} .
$$

With respect to this action, we have the zero degree cohomology group, denoted by $H^{0}(g(b), G)$. The following proposition is immediate.

2.3.2. Proposition. Let $b, g(b)$ be defined as above. Then there is a one-toone correspondence between $H^{0}(g(b), G)$ and the subgroup of elements $d$ in $G$ commuting with $\theta_{b}$.

\section{HERMitian REPRESENTATIONS OF $\hat{g}$}

3.1. We will concentrate our effort in studying the cohomology $H^{1}(\hat{g}, G)$ in the special case when $G$ is the symplectic group $\operatorname{Sp}_{2 n}(\mathbb{Q})$, and $g$ consists of inner automorphisms. In this setting, the theory can be reduced to one of skew-hermitian representations of $\hat{g}$.

First of all, the center of $\mathrm{Sp}_{2 n}(\mathbb{Q})$ is a cyclic group of order 2 with $-I_{2 n}$ as the nontrivial generator. It follows that $\hat{g}$ is a 2 -fold covering over $g$ with a nontrivial element $\omega$ in the kernel of the projection $\hat{g} \rightarrow g$, which maps onto $-I_{2 n}$.

As a consequence of $\S 2$ there is a one-to-one correspondence between $H^{1}\left(\mathrm{~g}, \mathrm{Sp}_{2 n}(\mathbb{Q})\right)$ and the set of isomorphism classes of pairs $(V, \lambda)$ consisting of a $2 n$-dimensional vector space $V$ over $\mathbb{Q}$ and a pairing $\lambda: V \times V \rightarrow \mathbb{Q}$ satisfying the following conditions:

$\lambda$ is nonsingular and skew symmetric;

$$
\begin{aligned}
& \lambda(u \cdot g, v \cdot g)=\lambda(u, v) \text { for } u, v \in V, g \in \hat{g} ; \\
& v \cdot \omega=-v \text { for } v \in V .
\end{aligned}
$$

Given any $\hat{g}$-invariant pairing $(V, \lambda)$, we define a new pairing $h: V \times V \rightarrow$ $\mathbb{Q}[\hat{g}]$ by means of the formula: $h(u, v)=\sum \lambda(u \cdot g, v) g^{-1}(g \in \hat{g})$. Then $h$ has the following properties:

$$
\begin{aligned}
& h \text { is nonsingular and skew-hermitian, } h(u, v)=-l h(v, u) \\
& \text { with respect to the involution } l: \sum a_{g} g \mapsto \sum a_{g} g^{-1} ;
\end{aligned}
$$

$$
h(u \cdot g, v)=h(u, v) g \text { for } u, v \in V, g \in \hat{g} .
$$

(Note that $h(u, v \cdot g)=g^{-1} h(u, v)$.) Conversely, given a pairing $(V, h)$ satisfying (3.1.3)-(3.1.5), there is a corresponding $\hat{g}$-invariant nonsingular skew symmetric pairing $(V, \lambda)$ defined by

$$
\lambda: V \times V \stackrel{h}{\rightarrow} \mathbb{Q}(\hat{g}) \stackrel{\text { tr }}{\rightarrow} \mathbb{Q}, \quad \text { where } \operatorname{tr}\left(\sum a_{g} g\right)=a_{1} .
$$

It is easy to verify that the above two constructions $(V, \lambda) \rightarrow(V, h),(V, h) \rightarrow$ $(V, \operatorname{tr} \circ h)$ are the inverse of each other. 
3.1.6. Theorem. Under the assumption that $g$ operates on $\mathrm{Sp}_{2 n}(\mathbb{Q})$ by inner automorphisms, the cohomology $H^{1}\left(\mathrm{~g}, \mathrm{Sp}_{2 n}(\mathbb{Q})\right)$ is the same as the set of isomorphism classes of nonsingular, skew-hermitian, $\mathbb{Q}[\hat{g}]$-pairings $(V, h)$ satisfying (3.1.3)-(3.1.5).

Furthermore given a cohomology class $b$ in $H^{1}\left(g, \operatorname{Sp}_{2 n}(\mathbb{Q})\right)$, the zero degree cohomology group $H^{0}\left(g(b), \mathrm{Sp}_{2 n}(\mathbb{Q})\right)$ is the same as the subgroup of centralizers $\hat{\rho}[\hat{g}(b)]$ in $\mathrm{Sp}_{2 n}(\mathbb{Q})$. Expressed in the terminology in the previous paragraph, this amounts to the $\mathbb{Q}[\hat{g}]$-automorphisms of $V$ preserving the skew-hermitian pairing $(V, h)$.

3.1.7. Theorem. Let $b$ be a cohomology class in $H^{1}\left(g, \mathrm{Sp}_{2 n}(\mathbb{Q})\right)$ and let $(V, h)$ be the corresponding hermitian $\mathbb{Q}[\hat{g}]$-pairing as in (3.1.6). Then the cohomology group $H^{0}\left(g(b), \mathrm{Sp}_{2 n}(R)\right)$ is isomorphic to the unitary group $U(V, h)$.

3.2. The procedure of classifying all the pairs $(V, h)$ satisfying (3.1.3)(3.1.5) is well known (see, for instance Satake [7, §IV-2]).

We decompose the $\mathbb{Q}[\hat{g}]$-module $V$ into its primary components-

$$
V=\bigoplus V[\chi]
$$

where each $V[\chi]$ is a direct sum of $n[\chi]$ copies of an irreducible $\mathbb{Q}[\hat{g}]$-module $W[\chi]$ and $\chi$ is the character of an irreducible representation of $\hat{g}$. In particular this means that

$$
\sum n[\chi] \operatorname{dim}_{\mathbb{Q}} W[\chi]=\operatorname{dim}_{\mathbb{Q}} V .
$$

Define the "twisted" group algebra $\mathbb{Q} \omega[g]$ to be the quotient of $\mathbb{Q}[\hat{g}]$ by the two-sided ideal $\langle 1+\omega\rangle$ generated by $1+\omega$. Then, from the Wedderburn theorem, the group algebra $\mathbb{Q}[\hat{g}]$ is a direct product of $\mathbb{Q}[g]$ and $\mathbb{Q} \omega[g]$. As is well known, the group algebra $\mathbb{Q}[\hat{g}]$ is a product of simple algebras $\operatorname{End}_{D[\chi]}(W[\chi])$, where $D[\chi]$ denotes the division algebra $\operatorname{End}_{\hat{\jmath}}(W[\chi])$. Thus there are two types of representations: those appear in the first factor $\mathbb{Q}[\mathcal{g}]$ and those appear in the second factor $\mathbb{Q}_{\omega}[\mathcal{g}]$. The first consists of representations for which $\omega$ operates trivially, and the second consists of representations for which $\omega$ operates as -1 . Thus for a $\hat{g}$-represenation $V$ to factor through $\mathbb{Q}_{\omega}[\mathrm{g}]$, it is necessary and sufficient that its multiplicities satisfies the following condition:

$$
\chi(\omega)>0 \Rightarrow n[\chi]=0 .
$$

Therefore the set of elements of $H^{1}(g, G L(V))$ which correspond to those representations of $\hat{g}$ such that $\omega$ operates as -1 is the same as the set of nonnegative integers $n[\chi]$ satisfying (3.2.2) and (3.2.3).

Next we turn to the problem of classifying to skew-symmetric $\hat{g}$-invariant pairings $\lambda: V \times V \rightarrow \mathbb{Q}$. It follows from Schur's lemma that any $\hat{g}$-invariant, bilinear pairing $(V, \lambda)$ is decomposed into an orthogonal sum of its primary components, $(V, \lambda)=\sum(V[\chi], \lambda[\chi])$, and so we can concentrate our discussion on the primary components $(V[\chi], \lambda[\chi])$. As a vector space over $\mathbb{Q}$, the $W[\chi]$ has at least one positive-definite, symmetric pairing $b(x, y)$, and by taking the average with respect to the group action we arrive at a $\hat{g}$-invariant, nonsingular, symmetric pairing $b$ over $W[\chi]$.

As is well known, any nonsingular symmetric pairing $(X, \beta)$ gives rise to an involution $l(\beta)$ on the endomorphism ring of $X$. For, from the nonsingular pairing, we have an adjoint isomorphism $\operatorname{ad}(\beta): X \rightarrow \operatorname{Hom}(X, \mathbb{Q})=$ 
$X^{*}$ between $X$ and its dual $X^{*}$. Given an endomorphism $\psi$ of $X$, there is an induced endomorphism on its dual $\psi^{*}: X^{*} \rightarrow X^{*}$. The composition $(\operatorname{ad} \beta)^{-1} \circ \psi^{*} \circ(\operatorname{ad} \beta)$ gives us a new endomorphism of $X$ which represents the image of $\psi$ under the above involution $l(\beta)$,

$$
l(\beta)(\psi)=(\operatorname{ad} \beta)^{-1} \circ \psi^{*} \circ(\operatorname{ad} \beta) .
$$

If $\lambda$ is a $\hat{g}$-invariant pairing, then $l(\lambda)$ preserves $\operatorname{End}_{\hat{g}}(V)$. Applying this situation to the irreducible representation $W[\chi]$ and the symmetric pairing $b$, we obtain an involution $l^{D}: D[\chi] \rightarrow D[\chi]$ on the division algebra $D[\chi]=$ End $_{\hat{g}}(W[\chi])$.

Let $U[\chi]$ be the vector space $\operatorname{Hom}_{\hat{g}}(W[\chi], V)$. Then $V[\chi]$ is isomorphic to the tensor product of $U[\chi]$ and $W[\chi]$ over $D[\chi]$. Then as shown in [7] there exists a skew-hermitian pairing $h^{W}: W[\chi] \times W[\chi] \rightarrow D[\chi]$ such that

$$
h^{W}(x, y \cdot d)=h^{W}\left(x \cdot l^{D}(d), y\right)
$$

for all $x, y$ in $W[\chi], d \in D[\chi]$. Furthermore there is a one-to-one correspondence between nonsingular, $\hat{g}$-invariant, skew-symmetric pairings $\lambda[\chi]: V[\chi] \times$ $V[\chi] \rightarrow \mathbb{Q}$ and nonsingular skew-hermitian pairings $h[\chi]: U[\chi] \times U[\chi] \rightarrow \mathbb{Q}$ such that

$$
\lambda[\chi]\left(u \times w, u^{\prime} \times w^{\prime}\right)=\operatorname{tr}_{D / Z(D)}\left(h[\chi]\left(u, u^{\prime}\right) \cdot l^{D}\left(h^{W}\left(w, w^{\prime}\right)\right)\right) .
$$

Let $H^{1}(U[\chi])$ denote the set of equivalent classes of skew hermitian, $D[\chi]-$ pairings over $U[\chi]$. Over a $\hat{g}$-vector space $\bigoplus U[\chi]$, the set of such skew hermitian, $\hat{g}$-pairings is given by a product

$$
H^{1}(\bigoplus U[\chi])=\prod H^{1}(U[\chi])
$$

3.2.4. Theorem. There exists a natural isomorphism of cohomology

$$
H^{1}\left(g, \operatorname{Sp}_{2 n}(\mathbb{Q})\right) \cong \bigcup H^{1}(\bigoplus U[\chi]),
$$

where the disjoint union on the right runs through all direct sum $\oplus U[\chi]$ such that

$$
\begin{gathered}
\sum n[\chi] \operatorname{dim} W[\chi]=2 n, \quad n[\chi]=\operatorname{dim} U[\chi], \\
\chi(\omega)>0 \Rightarrow n[\chi]=0 .
\end{gathered}
$$

Let $\hat{\rho}$ be a symplectic representation of $\hat{g}$ into $\operatorname{Sp}_{2 n}(\mathbb{Q})$, and let $h[\chi]=U[\chi] \times$ $U[\chi] \rightarrow D[\chi]$ be the skew-hermitian pairing defined as above. Then there exists a natural isomorphism

$$
H^{0}\left(g, \operatorname{Sp}_{2 n}(\mathbb{Q})\right) \cong \prod_{[\chi]} U(h[\chi])
$$

where $U(h[\chi])$ is the unitary group preserving the skew-hermitian pairing $h[\chi]=$ $U[\chi] \times U[\chi] \rightarrow D[\chi]$.

The part of the theorem about $H^{1}$ follows from the above discussion. We recall that the cohomology $H^{0}\left(g ; \mathrm{Sp}_{2 n}(\mathbb{Q})\right)$ is the same as the group of automorphisms preserving the $\hat{g}$-invariant, skew-symmetric pairing $(V, \lambda)$. From the orthonormal decomposition $(V, \lambda)=\sum(V[\chi], \lambda[\chi])$ we have $\operatorname{Aut}_{\hat{g}}(V, \lambda)=$ 
$\prod \operatorname{Aut}_{\hat{\mathrm{g}}}(V[\chi], \lambda[\chi])$, and from the tensor product decomposition above, we have $\operatorname{Aut}(V[\chi], \lambda[\chi]) \cong U(h[\chi])$. Thus we get (3.2.8).

\section{Finite group actions on Siegel modular SPACE}

4.1. Let $V$ be a rational vector space of dimension $2 n$, endowed with a nonsingular, skew-symmetric pairing $\lambda: V \times V \rightarrow \mathbb{Q}$. Denote by $\operatorname{Sp}(V)$ the group of automorphisms which preserves this pairing $(V, \lambda)$. We use the notation $\operatorname{Sp}\left(V_{\mathbb{R}}\right)$, where $V_{\mathbb{R}}$ is the real vector space $V \otimes \mathbb{R}$, to denote the real points.

Let $\mathfrak{G}(V)$ be the Siegel upper half space associated to $V \cdot \mathfrak{G}(V)$ is isomorphic to $K \backslash \operatorname{Sp}\left(V_{\mathbf{R}}\right)$ where $K$ is the maximal compact subgroup of $\operatorname{Sp}\left(V_{\mathbf{R}}\right)$. A point $z$ in $\mathfrak{G}(V)$ can be viewed as a complex structure $J_{z}$ on $V_{\mathbb{R}},\left(J_{z}: V_{\mathbb{R}} \rightarrow\right.$ $\left.V_{\mathbb{R}}, J_{z}^{2}=-\mathrm{id}\right)$ such that the bilinear pairing $(u, v) \mapsto \lambda\left(u, J_{z} v\right)$ is symmetric and positive definite.

Let $L$ be a lattice in $V$ such that the restriction of the pairing $\lambda$ to $L$ takes on integral values $\lambda: L \times L \rightarrow \mathbb{Z}$. Let $\left(x_{i}\right)$ denote a finite collection of points in $V$. We obtain an arithmetic subgroup $\Gamma$ in $\operatorname{Sp}(V)$ by considering all automorphisms $\psi$ in $\operatorname{Sp}(V)$ such that $\psi(L)=L, \psi\left(x_{i}\right) \equiv x_{i} \bmod L$,

$$
\Gamma=\left\{\psi \in \operatorname{Sp}(V) \mid \psi(L)=L, \psi\left(x_{i}\right) \equiv x_{i} \bmod L\right\} .
$$

This subgroup operates properly discontinuously on the Siegel upper half space $\mathfrak{G}(V)$ and its quotient space $\mathfrak{G}(V) / \Gamma$ is a Siegel modular space.

Let $D$ be a product a division algebras over $\mathbb{Q}$ with a positive involution $l^{D}$, and let $\Psi$ be a representation of $D$ into $\operatorname{End}(V)$. We refer to [9] for the definition of PEL structures on abelian varieties. The set of all isomorphism classes of PEL structures of a fixed type $\Omega=\left(D, \Psi, l^{D} ; V, \lambda, L ; x_{i}\right)$ forms a moduli space. We refer this moduli space as the Shimura variety and denote it by $S_{\Psi}$.

Let

$$
\begin{aligned}
& G_{\Psi}=\{g \in \operatorname{Sp}(V) \mid g \cdot \Psi(\alpha)=\Psi(\alpha) \cdot g, \alpha \in D\}, \\
& \mathfrak{G}_{\Psi}=\{(V, J) \in \mathfrak{G}(V) \mid J \cdot \Psi(\alpha)=\Psi(\alpha) \cdot J, \alpha \in D\} .
\end{aligned}
$$

Then according to Shimura [9-11] there is a natural isomorphism between the moduli space $S_{\Psi}$ and the quotient space $\mathfrak{G}_{\Psi} / \Gamma \cap G_{\Psi}$.

4.2. Proposition. Let $g$ be a finite group of automorphism of $\operatorname{Sp}\left(V_{\mathbb{R}}\right)$ which keeps the maximal compact subgroup $K$ and the arithmetic subgroup $\Gamma$ invariant. Then every connected component of the set $(\mathfrak{G}(V) / \Gamma)^{\&}$ of fixed points of $g$ in $\mathfrak{G}(V) / \Gamma$ is a Shimura variety.

4.3. The proposition is an immediate consequence of 1.4. However we can give a more explicit description in terms of the characters of $\hat{g}$. The action of $\hat{g}$ on the symmetric space $\mathfrak{G}(V)$ must have a fixed-point. Let $z=\left(V_{\mathbb{R}}, J_{z}\right)$ be such a fixed-point in $\mathfrak{G}(V)$. Let $A$ be the abelian variety $V_{\mathbb{R}} / L$ with complex structure $J_{z}$. Since $\alpha J_{z}=J_{z} \alpha,(\alpha \in \mathbb{Q}[g])$, there is a homomorphism

$$
\mathbb{Q}[g] \rightarrow \operatorname{End}_{\mathbb{Q}}(A)
$$

of the group algebra $\mathbb{Q}[g]$ into the endomorphism algebra $\operatorname{End}_{\mathbb{Q}}(A)$ of $A$.

Decompose $V$ into primary components $V=\bigoplus V\left[\chi_{i}\right] \quad(1 \leq i \leq l)$ as in (3.2.1). Let $D\left[\chi_{i}\right]$ be the division algebra $\operatorname{End}_{\hat{g}}\left(W\left[\chi_{i}\right]\right)$ and $D$ be the product 
of all the division algebras $D\left[\chi_{i}\right], D=D\left[\chi_{i}\right] \times \cdots \times D\left[\chi_{l}\right]$. Then, from Satake [7, Lemma IV.1.1], the composite

$$
D \rightarrow \mathbb{Q}[\hat{g}] \rightarrow \operatorname{End}_{\mathbb{Q}}(V)
$$

gives us an injection of $D$ as a subalgebra in $\operatorname{End}_{\mathbb{Q}}(V)$. Using (4.3.1) we get a monomorphism $\Psi: D \hookrightarrow \operatorname{End}_{\mathbb{Q}}(A)$.

Since $\lambda$ is $\hat{g}$-invariant, we have the selfadjoint condition

$$
\lambda\left(\left(\sum a_{g} \cdot g\right) \cdot x, y\right)=\lambda\left(x,{ }^{\prime}\left(\sum a_{g} \cdot g\right) \cdot y\right)
$$

satisfied for all the elements in the group algebra $\mathbb{Q}[\hat{g}]$. This preserves the natural involution $l^{D}$ on $D$ and on the endomorphism algebra $\operatorname{End}_{\mathbb{Q}}(A)$. By the solution to the congruence subgroup problems there exists a level structure $x_{i}$ such that $\Gamma$ is a congruence subgroup preserving this level structure.

Let $S_{\Psi}$ be the Shimura variety of the type $\left(D, \Psi, l^{D} ; V, \lambda, L ; x_{i}\right)$, and let $G_{\Psi}$ and $\mathfrak{G}_{\Psi}$ be defined as in $\S 4.1$. From representation theory, it is known that the automorphisms of $\rho=(V, \lambda)$ which preserve the $g$-action are precisely those automorphisms which preserve the $D$-action,

$$
\operatorname{Aut}_{\mathcal{g}}(V, \lambda) \cong \operatorname{Aut}_{D}(V, \lambda) \text {. }
$$

Hence the two subgroups $H^{0}\left(g, \mathrm{Sp}_{2 n}(V)\right)$ and $G_{\Psi}$ in $\mathrm{Sp}(V)$ are the same. Consider the orbit of the $z=\left(V, J_{z}\right)$ under the action of this group. On the one hand, it is the fixed-point subspace $\mathfrak{G}(V)^{\mathscr{f}}$ and on the other hand it is the bounded symmetric domain $\mathfrak{G}_{\Psi}$. Therefore they must coincide $\mathfrak{G}(V)^{\mathscr{F}} \cong \mathfrak{G}_{\Psi}$, and the same holds for their arithmetic quotients

$$
F(\rho) \cong \mathfrak{G}_{\Psi}, \quad[\rho] \in H^{1}(g, \operatorname{Sp}(V)) .
$$

This proves that the connected component $F(\rho)$ in the fixpoint set $(\mathfrak{G}(V) / \Gamma)^{\mathscr{\rho}}$ is a Shimura variety. As for the other components $F(b)$, we only have to replace $g$ by the twisted action $g(b)$, and repeat the same argument as above.

4.4. In [9], Shimura studied the nature of these varieties according to the classification of the algebra $D$. It is interesting to compare his classification with the analysis in $\S 3$. For this, it is enough to study only the situation when $D$ is a division algebra. Division algebras with positive involution separate into types I, II, III, IV, and we have the corresponding four types of $S_{\Psi}$. We shall omit details, instead, we give two examples.

Example (4.4.1). Let $g$ be the cyclic group of order $p$ where $p$ is an odd prime. Since $H^{2}(g ; \mathbb{Z} / 2)=0$ any central extension $\hat{g}$ of $g$ is a trivial central extension. Hence $\hat{g}$ is a product of $g$ and a cyclic group of order $2, g \cong$ $g \times \mathbb{Z} / 2$, and the group algebra, $\mathbb{Q}[\hat{g}]$ is also a product

$$
\mathbb{Q}[\hat{g}] \simeq \mathbb{Q}[\mathscr{g}] \times \mathbb{Q} \omega[\mathcal{g}], \quad \mathbb{Q} \omega[\mathscr{g}] \simeq \mathbb{Q}\left[e^{2 \pi i / p}\right] \times \mathbb{Q} .
$$

Suppose the representation of $\hat{g}$ in $\operatorname{Sp}(V)$ have no trivial factor. Then the algebra $D$ coincides with the cyclotomic field $\mathbb{Q}\left[e^{2 \pi i / p}\right]$ and the involution is the complex conjugation. In other words, we are in (Type IV) with $p-1$ isomorphisms of $\mathbb{Q}\left[e^{2 \pi i / p}\right]$ into the complex field

$$
\tau_{1}, \ldots, \tau_{\frac{p-1}{2}}, \bar{\tau}_{1}, \ldots, \bar{\tau}_{\frac{p-1}{2}} \text {. }
$$


Since $\Psi$ is of degree $n$, we have

$$
n=m(p-1), \quad r_{v}+s_{v}=m, \quad v=1, \ldots, \frac{p-1}{2}
$$

and $G_{\Psi}$ is a product of unitary groups. In particular if $m=1$, we have either $r_{v}$ or $s_{v}$ is 0 and either case the fixed-point set consists of isolated points. If $m=1$, then we have three possibilities: (i) $r_{v}=2, s_{v}=0$, (ii) $r_{v}=0, s_{v}=2$, (iii) $r_{v}=1, s_{v}=1$. In the first two cases, we have isolated points as fix point set, and in the third case we have a product of curves as its fixpoint set.

Example (4.4.2). The situation for the cyclic group of order two is similar. In the case when $\hat{g}$ is a split extension, $\hat{g} \cong g \times \mathbb{Z} / 2$, we have the group algebra

$$
\mathbb{Q}_{\omega}[g] \cong \mathbb{Q}[g] \cong \mathbb{Q}_{+} \times \mathbb{Q}_{-}
$$

and so we are in Type I situation. The fixed-point components are a product of Siegel modular spaces of various dimensions.

\section{REFERENCES}

1. A. Borel and J. Tits, Homomorphismes "abstraits" de groupes algébriques simples, Ann. of Math (2) 97 (1973), 499-571.

2. E. Gottschiling, Über die Fixpunkte der Siegelschen Modulgruppe, Math. Ann. 143 (1961), 111-149.

3. __ Über der Fixpunktergruppe die Siegelschen Modulgruppe, Math. Ann. 143 (1961), 399-430.

4. S. Helgason, Differential geometry and symmetric spaces, Academic Press, 1962.

5. M. Kneser, Lectures on Galois cohomology of classical groups, Tata Institute of Fundamental Research, Bombay, 1969.

6. J. Rohlfs, The Lefschetz number of an involution on the space of classes of positive definite quadratic forms, Comment. Math. Helv. 56 (1981), 272-296.

7. I. Satake, Algebraic structures of symmetric domains, Princeton Univ. Press, 1980.

8. J. P. Serre, Cohomologie Galoisienne, Lecture Notes in Math., vol. 5, Springer-Verlag, 1965.

9. G. Shimura, Moduli of abelian varieties and number theory, Proc. Sympos. Pure Math., vol. 9, Amer. Math. Soc., Providence, R.I., 1966, pp. 312-332.

10. On analytic families of polarized abelian varieties and automorphic functions, Ann. of Math. (2) 78 (1963), 149-192.

11. __ Moduli and fibre systems of abelian varieties, Ann. of Math. (2) 83 (1966), 294-338.

12. T. A. Springer, Galois cohomology of linear algebraic groups, Proc. Sympos. Pure Math., vol. 9, Amer. Math. Soc., Providence, R.I., 1966, pp. 149-158.

13. A. Weil, Algebras with involutions and the classical groups, J. Indian Math. Soc. 24 (1960), 589-623.

Department of Pure Mathematics, University of Sydney, Sydney NSW 2006, AusTRALIA

Department of Mathematics, Yale University, New Haven, Connecticut 06520 